\title{
ION-STREAMING INSTABILITIES: ELECTROMAGNETIC AND ELECTROSTATIC
}

BY

F, W. PERKINS

\section{PLASMA PHYSICS LABORATORY}

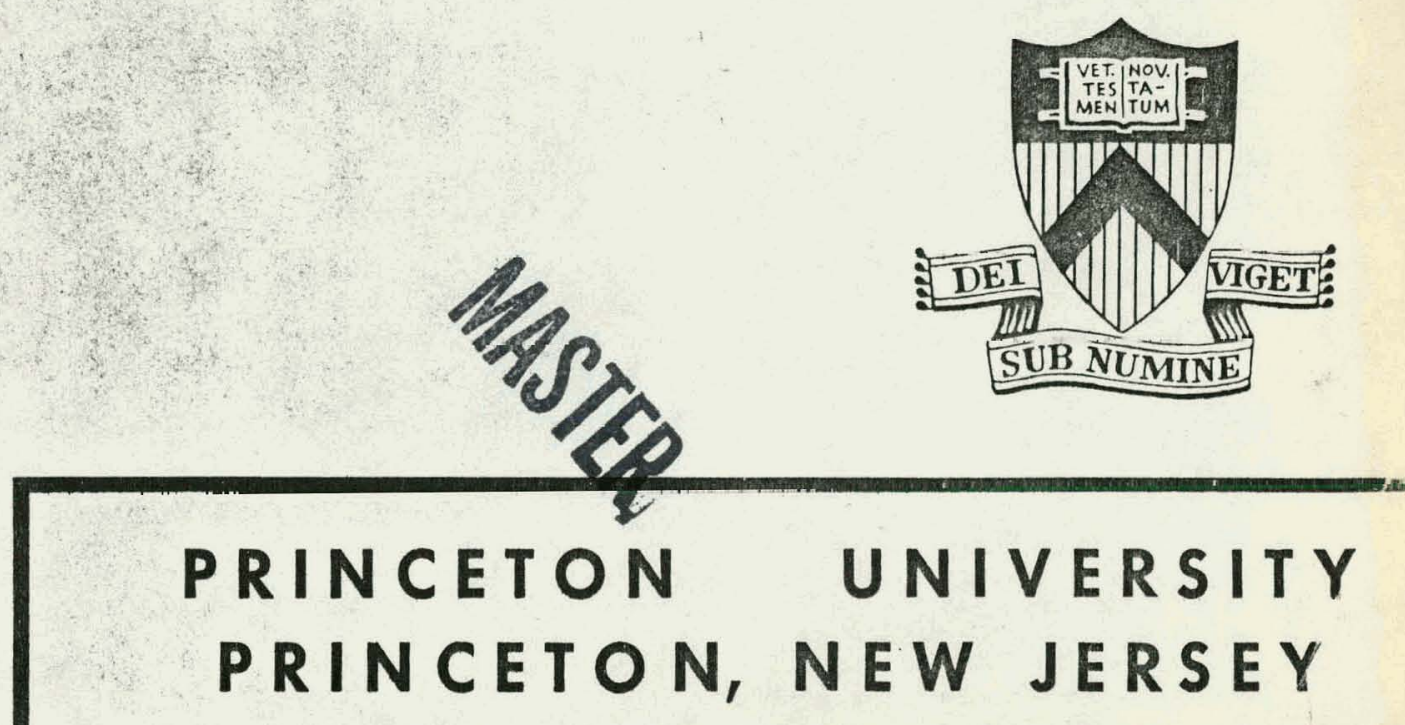

This work was supported by U. S. Atomic Energy Commission Contract AT $(11-1)-3073$. Reproduction, translation, publication, use, and disposal, in whole or in part, by or for the United States Government is permitted. 


\section{DISCLAIMER}

This report was prepared as an account of work sponsored by an agency of the United States Government. Neither the United States Government nor any agency Thereof, nor any of their employees, makes any warranty, express or implied, or assumes any legal liability or responsibility for the accuracy, completeness, or usefulness of any information, apparatus, product, or process disclosed, or represents that its use would not infringe privately owned rights. Reference herein to any specific commercial product, process, or service by trade name, trademark, manufacturer, or otherwise does not necessarily constitute or imply its endorsement, recommendation, or favoring by the United States Government or any agency thereof. The views and opinions of authors expressed herein do not necessarily state or reflect those of the United States Government or any agency thereof. 


\section{DISCLAIMER}

Portions of this document may be illegible in electronic image products. Images are produced from the best available original document. 
Ion-Streaming Instabilities: Electromagnetic and Electrostatic

F. W. Perkins

Plasma Physics Laboratory, Princeton University

Princeton, New Jersey 08540.

ABSTRACT

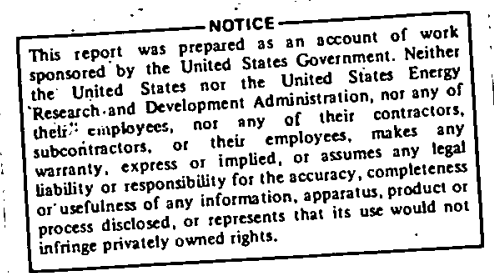

Electromagnetic effects are shown to reduce the threshold velocity (relative to electrostatic calculation) for ion-beam instabilities in plasmas where the beam velocity, taken parallel to the magnetic field, becomes a modest fraction of the Alfvén velocity. The most unstable mode occurs for frequencies in the ion-cyclotron frequency range and is caused by an interaction of the beam with a type of clectromagnetic ion-cyciotron wave. Applications of the theory range from basic research experiments, to collisionless shocks and twocomponent thermonuclear burning devices. 


\section{INTRODUCTION}

Ion streaming along magnetic fields as a source of free energy to drive plasma instabilities in germane to the problem of parallel magnetic shocks, 1 to basic laboratory studies of plasma instabilities, ${ }^{2}$ and to two-component thermonuclear plasmas $^{3}$ when injected beams provide the main source of thermonuclear burning. Several calculations ${ }^{4-6}$ have demonstrated that ion-beam instabilities in magnetic field-free plasmas can be stabilized by $\mathrm{T}_{\mathrm{e}} / \mathrm{T}_{\mathrm{b}}<2$, where $\mathrm{T}_{\mathrm{b}}$ is the "temperature" associated with the spreads in beam energy and direction. Four theoretical studies address themselves to streaming in the presence of a magnetic field: Weibel ${ }^{7}$ derives an instability criterion for electrostatic instabilities, while Golden, Linson, and Mani ${ }^{1}$ treat the stability of electromagnetic waves propagating parallel to the magnetic field. They conclude that such waves are unstable if the parallel velocity exceeds $2 v_{A}$, where $v_{A} \equiv\left(B^{2} / 4 \pi n e^{M}\right)^{1 / 2}$ denotes the Alfvén velocity. The stability analyses of Stix, ${ }^{8}$ and of Berk et al. ${ }^{9}$ for two-component tokamak plasmas do not consider beam instabilities for electromagnetic ion-cyclotron waves. On the experimental side, the paper of Ishizuka, ono, and Kojima ${ }^{2}$ has shown that electrostatic ioncyclotron waves can be made unstable by ion beams with streaming velocities $U$ satisfying $c_{s} \ll U \ll v_{e}, v_{A}$ where $c_{S}$ is the sound speed and $v_{e}=\left(2 \mathrm{~T}_{\mathrm{e}} / \mathrm{m}\right)^{1 / 2}$.

This paper examines the linear stability of both electrollagnetic and electrostatic ion-cyclotron waves in plasmas 
containing ion beams. Ion-cyclotron waves have the property that their parallel phase velocity $\omega / k \|$ lies in the range $c_{s}<\omega / k_{\|}<v_{e}$ where they can interact with ion beams. For the electromagentic ion-cyclotron wave in particular, the phase velocity is below the Alfvén velocity and consequently it may become unstable when the streaming velocity is below the Alfvén speed. Similar phase velocity arguments apply to instabilities generated by electron current. 10 we shall first present our model and dispersion relation, then discuss the beam instabilities resulting from strong, interpenetrating ion beams. The paper proceeds to the case where the beam is not strong and the instabilities have a resonant-particle velocity-space character. We conclude with a discussion of how ion-cyclotron instabilities might arise in a two-component thermonuclear burning experiment and in basic research devices.

\section{MODEL AND DISPERSION RELATION}

The starting point is a model of a uniform low- $\beta$ plasma containing a constant magnetic field in the $\underset{\mathrm{z}}{\hat{z}}$-direction. Figure 1 illustrates our three-component model of the plasma. It consists of: Maxwellian electrons with a temperature $\mathrm{T}_{e}$, density $\mathrm{n}_{e}$, and no net velocity; a cold ion component with temperature $T_{C}$ and density $n_{C}$ also at rest; and two oppositelydirected beams of density $n_{b}$, streaming velocity $U$, and velocity spread characterized by the temperature $\mathrm{T}_{\perp}, \mathrm{T}_{\|} \cdot \mathrm{All}$ species are singly charged so that $n_{e}=n_{c}+2 n_{b}$. 
We shall examine waves propagating almost perpendicular to the magnetic field in the $x-z$ plane with a perpendicular wave number satisfying $\rho_{-}^{-2} \geq \mathrm{k}_{1}^{2}>\mathrm{c}^{2} / \omega_{\mathrm{pi}}^{2} \sim \beta \rho^{-2}$ where $\rho^{2} \equiv \mathrm{T}_{1} / \mathrm{M} \Omega^{2}$. In these circumstances, $E_{y}$ is negligible and the full electromagnetic wave equations become

$$
\begin{gathered}
\left(\frac{\omega^{2}}{c^{2}} k_{x x}-k_{\|}^{2}\right) E_{x}+\left(k_{\perp} k_{\|}+\frac{\omega^{2}}{c^{2}} k_{x z}\right) E_{\|}=0 \\
\left(k_{\perp} k_{\|}+\frac{\omega^{2}}{c^{2}} k_{z x}\right) E_{x}+\left(-k_{\perp}^{2}+\frac{\omega^{2}}{c^{2} \cdot k_{\|}^{2} D_{e}^{2}} Y_{0}^{(e)}+\frac{\omega^{2}}{c^{2}} k_{z z}\right) E_{\|}=0
\end{gathered}
$$

where the tensor $\underset{\sim}{\mathrm{K}}$ describes the ion dynamics and is given in terms of the ion distribution function $f_{0}$ by ${ }^{11}$

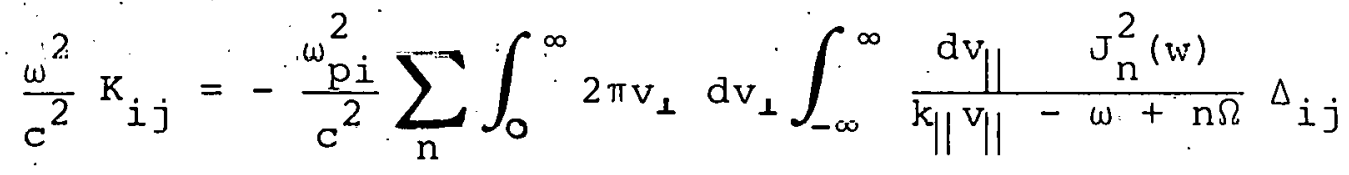

where $\mathrm{w}=\mathrm{k}_{\perp} \mathrm{v}_{\perp} / \Omega$, and $: \quad \mathrm{i}$

$$
\begin{aligned}
& \Delta_{x x}=\frac{n^{2} \Omega^{2}}{k_{\perp}^{2} v_{\perp}} \cdot\left[\omega \frac{\partial f_{0}}{\partial v_{\perp}}+k_{\|}\left(\frac{\partial f_{0}}{\partial v_{\|}} v_{\perp}-\frac{\partial f_{0}}{\partial v_{\perp}} v_{\|}\right)\right] \\
& \Lambda_{\mathrm{XZ}}=\frac{\mathrm{n} \Omega}{\mathrm{k}_{\perp}} \quad\left[\omega \frac{\partial \mathrm{f}_{\mathrm{O}}}{\partial \mathrm{v}_{\|}}-\mathrm{n} \Omega\left(\frac{\partial \mathrm{f}_{\mathrm{O}}}{\partial \mathrm{v}_{\|}}-\mathrm{v}_{\mathrm{v}_{\perp}} \frac{\partial \mathrm{f}_{\mathrm{O}}}{\partial \mathrm{v}_{\perp}}\right)\right]
\end{aligned}
$$




$$
\begin{gathered}
\Delta_{z x}=\frac{v_{\|} \|^{\Omega n}}{k_{\perp} v_{\perp}}\left[\omega \frac{\partial f_{0}}{\partial v_{\perp}}+k_{\|}\left(\frac{\partial f}{\partial v_{\|}} v_{\perp}-\frac{\partial f}{\partial v_{\perp}} v_{\|}\right)\right] \\
\Delta_{z z}=v_{\|}\left[\omega \frac{\partial f}{\partial v_{\|}}-n \Omega\left(\frac{\partial f}{\partial v_{\|}}-\frac{v_{\|}}{v_{\perp}} \cdot \frac{\partial f}{\partial v_{\perp}}\right)\right]
\end{gathered}
$$

The electrons enter through $\mathrm{Y}_{\mathrm{O}}^{(\mathrm{e})}$

$$
\mathrm{Y}_{0}^{(\mathrm{e})}=\int_{-\infty}^{\infty} \cdot d v_{\|} \frac{\mathrm{k}_{\|} \mathrm{v}_{\|}}{\mathrm{k}_{\|} \mathrm{v}_{\|}-\omega}\left(\frac{\mathrm{m}}{2 \pi \mathrm{T}}\right)^{1 / 2} \exp \left(-\frac{\mathrm{mv} \|^{2}}{2 \mathrm{~T}_{\mathrm{e}}}\right)
$$

The dispersion relation is obtained by setting the determinant of the coefficient of $(1-2)$ equal to zero:

$$
\begin{gathered}
\frac{\omega^{2}}{c^{2}}\left[k_{\perp}^{2} k_{x x}+k_{\perp} k_{\|}\left(k_{x z}+k_{z x}\right)+k_{\|}^{2} k_{z z}^{\prime}\right] \\
=\left(\frac{\omega^{2}}{c^{2} k_{\|}^{2} D_{e}^{2}} y_{0}^{(e)}+\frac{\omega^{2}}{c^{2}} k_{z z}^{(0)}\right)\left(\frac{\omega^{2}}{c^{2}} k_{x x}-k_{\|}^{2}\right) \\
+\frac{\omega^{4}}{e^{4}}\left(k_{x x} k_{z z}^{\prime}-k_{x z} K_{z x}\right)
\end{gathered}
$$

In (9), $K_{z z}$ has been split into two parts.

$$
K_{z z}=K_{z z}^{(0)}+K_{z z}^{\prime}
$$


where

$$
\begin{aligned}
& \frac{\left.{ }^{2}\right)^{2}}{c^{2}} \mathrm{~K}_{\mathrm{zz}}^{(0)}=-\frac{\omega_{\mathrm{pi}}^{2}}{c^{2}} \cdot \int \mathrm{dv}_{\sim_{\perp}} \int_{-\infty}^{\infty} \frac{d v_{\|} \mathrm{J}_{\mathrm{o}}^{2}(w)}{\mathrm{k}_{\|} v_{\|}-\omega} \omega v_{\|} \frac{\partial f_{0}}{\partial v_{\|}} \\
& =-\frac{\omega^{2}}{c^{2}} \frac{\omega_{\mathrm{p} i}^{2}}{\mathrm{k}_{\|}^{2}} \cdot \int \mathrm{dv}_{\perp} \int_{-\infty}^{\infty} \frac{d v_{\|} \mathrm{J}_{o}^{2}(w)}{\mathrm{k}_{\|} v_{\|}-\omega} \mathrm{k}_{\|} \frac{\partial \mathrm{f}_{\mathrm{o}}}{\partial v_{\|}}
\end{aligned}
$$

and

$$
\begin{gathered}
\frac{\omega^{2}}{c^{2}} k_{z z}^{\prime}=-\frac{\omega_{p i}^{2}}{c^{2}} \sum_{n} \int_{v_{\perp}} \int_{-\infty}^{\infty} d v_{\|} \frac{J_{n}^{2}(w) v_{\|}}{k_{\|} v_{\|}-\omega+n \Omega} \\
\therefore\left[\omega \frac{\partial f_{0}}{\partial v_{\|}}-n \Omega\left(\frac{\partial f}{\partial v_{\|}}-\frac{v_{\|}}{v_{\perp}} \frac{\partial f}{\partial v_{\perp}}\right)\right] \vdots
\end{gathered}
$$

Following standard procedures for electrostatic waves, ${ }^{11}$ the terms on the left hand side of (9) can be combined to give

$$
\begin{gathered}
\frac{\omega^{2}}{c^{2}}\left[k_{\perp}^{2} k_{x x}+k_{\perp} k_{\|}\left(k_{x z}+k_{z x}\right)+k_{\|}^{2} k_{z z}^{\prime}\right] \\
=-\frac{\omega^{2} \omega_{p i}^{2}}{c^{2}} \sum_{n} \int_{n} d_{\sim} \int_{-\infty}^{\infty} d v_{\|} \frac{J_{n}^{2}(w)}{k_{\|} v_{\|}-(1)+n \Omega}\left(k_{\|} \frac{\partial f_{o}}{\partial v_{\|}}+\frac{n \Omega}{v_{\perp}} \frac{\partial f_{o}}{\partial v_{\perp}}\right) \\
=\frac{\omega^{2} k_{\perp}^{2} \omega_{p i}^{2}}{c^{2} \Omega^{2}}
\end{gathered}
$$


which defines the nondimensional function $\theta$.

Let us now turn our attention to the terms quadratic in $\mathrm{K}$ on the right-hand side of (9). These can be written as

$$
\frac{w_{p i}}{c^{4}} \sum_{n, n} \int_{n} d v_{\perp}^{\prime} \cdot d v_{\perp} \int_{-\infty}^{\infty} d v_{\|} d v_{\|}^{\prime} \frac{J_{n}^{2}\left(w^{\prime}\right) J_{n}^{2}(w)}{\left(k_{\|} v_{\|}-w+n \Omega\right)\left(k_{\|} v_{\|}-w+n \Omega\right)}
$$

- $\frac{\Omega^{2}}{k_{\perp}^{2}}\left\{v_{\|}^{\prime}{ }^{2}\left[\frac{\omega}{v_{\perp}} \frac{\partial f_{0}}{\partial v_{\perp}}+k_{\|}\left(\frac{\partial f_{0}}{\partial v_{\|}}-\frac{v_{\|}}{v_{\perp}} \frac{\partial f_{0}}{\partial v_{\perp}}\right)\right]\left[\omega \frac{\partial f_{0}^{\prime}}{\partial v_{\|}^{\prime}}-n^{\prime} \Omega\left(\frac{\partial f}{\partial v_{\|}}-\frac{v_{l}^{\prime}}{v_{\perp}} \frac{\partial f_{0}}{\partial v_{\perp}^{\prime}}\right)\right]\right.$

$-n n^{\prime} v_{\|}^{\prime}\left[\omega \frac{\partial f_{0}}{\partial v_{\|}}-n \Omega\left(\omega \frac{\partial f_{0}}{\partial v_{\|}}-\frac{v_{\|}}{v_{\perp}} \frac{\partial f_{0}}{\partial v_{1}}\right)\right]\left[\frac{\omega}{v_{\perp}^{\prime}} \frac{\partial f_{0}}{\partial v_{1}^{\prime}}+k_{\mu}\left(\frac{\partial f_{0}}{\partial v_{\|}^{\prime}}-\frac{v_{1}^{\prime}}{v_{\perp}^{\prime}} \frac{\partial f_{0}}{\partial v_{\perp}^{\prime}}\right)\right]$

$=\frac{\omega_{p i}^{4}}{c^{4}} \sum_{n, n} \int_{d v_{\perp}^{\prime}} d v_{\perp} \int_{-\infty}^{\infty} \frac{d v_{\|} d v_{\|}^{\prime} J_{n}^{2}\left(w^{\prime}\right) J_{n}^{2}(w) \Omega^{2}}{\left(k_{\|} v_{\|}-\omega+n \Omega\right)\left(k_{\|} v_{\|}^{\prime}-\omega+n^{\prime} \Omega\right) k_{\perp}^{2}}$

$$
\begin{gathered}
\cdot \omega^{\prime}\left(v_{\|}{ }^{\prime}-n v_{\|}^{\prime}\right)\left[\omega \frac{\partial f_{0}}{\partial v_{\|}} \frac{1}{v_{\perp}^{\prime}} \frac{\partial f_{0}^{\prime}}{\partial v_{\perp}^{\prime}}\right. \\
\left.\ddots\left(k_{\|} \frac{\partial f_{0}}{\partial v_{\|}}+\frac{n \Omega}{v_{\perp}} \frac{\partial f_{0}}{\partial v_{\perp}}\right)\left(\frac{\partial f_{0}}{\partial v_{\|}^{\prime}}-\frac{v_{1}^{\prime}}{v_{\perp}^{\prime}} \frac{\partial f_{0}^{\prime}}{\partial v_{\perp}^{\prime}}\right)\right]
\end{gathered}
$$

where some interchanges between primed and unprimed variables of integration and summation were used in obtaining the right-hand side. The next manipulations utilize operations such as 


$$
\begin{aligned}
& \frac{\mathrm{v}_{\|} \mathrm{n}^{\prime} \therefore}{\mathrm{k}_{\|} \mathrm{v}_{\|}-\omega+\mathrm{n} \Omega}=\frac{\mathrm{n}^{i}}{\mathrm{k}_{\|}}\left(\frac{\omega-\mathrm{n} \Omega}{\mathrm{k}_{\|} \mathrm{v}_{\|}-\omega+\mathrm{n} \Omega}+\mathrm{l}\right) \\
& \rightarrow \frac{n !(\omega-\mathrm{n} \Omega)}{\mathrm{k}_{\|}\left(\mathrm{k}_{\|} \mathrm{v}_{\|}-\omega+\mathrm{n} \Omega\right)}
\end{aligned}
$$

with the unity: term vanishing in the summations and integrations. One then obtains

$$
\begin{aligned}
& \operatorname{tin} 6: 2=\text {. A } \\
& \underset{c}{\frac{\omega^{4}}{4}}\left(K_{x x} K_{z z}^{\prime}-K_{x z} K_{z x}\right)=
\end{aligned}
$$$$
=\frac{\omega_{p i}^{4} \omega^{2} \Omega^{2}}{c^{4} k_{1} k_{1}^{2}} \sum_{n, n}^{\prime} \int d v_{\perp} v_{\nu_{1}} \int_{-\infty}^{\infty} d v_{\|} d v_{\|}^{\prime} \frac{J_{n}^{2}(w) J_{n}^{2} i\left(w^{\prime}\right)}{\left(k_{\|} v_{\|}-\omega+n \Omega\right)\left(k_{\|} v_{\|}^{\prime}-\omega+n^{\prime} \Omega\right)}
$$$$
\text { - } n^{\prime}\left(n^{\prime}-n\right)\left\{\left[\omega \frac{\partial f_{0}}{\partial v_{\|}}-n \Omega\left(\frac{\partial f_{0}}{\partial v_{\|}}-\frac{v_{1}}{v_{\perp}} \frac{\partial f_{0}}{\partial v_{\perp}}\right)\right] \frac{1}{v_{\perp}} \cdot \frac{\partial f_{0}}{\partial v_{\perp}^{\prime}}\right.
$$

$$
\left.+k_{\|} \frac{\partial f_{0}}{\partial v_{\|}}\left(\frac{\partial f_{0}}{\partial v_{\|}}-\frac{v_{\|}^{\prime}}{v_{\perp}} \frac{\partial f_{0}}{\partial v_{i}}\right)\right\} \equiv k_{\perp}^{2} \frac{\omega_{p i}^{2} \omega^{2}}{c^{2} \Omega^{2}} \Psi
$$

which serves as a definition of the nondimensional function $\Psi$. Canceling out the common factor of $\omega^{2} / c^{2}$, one obtains the dispcrsion relalion 


$$
\frac{k_{\perp}^{2} \omega_{p i}^{2}}{\Omega^{2}}(\theta-\psi)=\left(\frac{1}{D_{e}^{2}} Y_{0}^{(e)}+k_{\|}^{2} K_{z z}^{(0)}\right)\left(\frac{\omega^{2} K_{x x}}{c^{2} k_{\|}^{2}}-1\right)
$$

From (13), one obtains the expression for $\theta$.

$$
\begin{aligned}
& \theta=-\sum_{n} \frac{\Omega^{2}}{k_{\perp}^{2}} \int d v_{\perp} \int_{-\infty}^{\infty} \frac{d v_{\|} \cdot J_{n}^{2}(w)}{k_{\|} v_{\|}-\omega+n \Omega}\left(k_{\|} \frac{\partial f_{0}}{\partial v_{\|}}+\frac{n \Omega}{v_{\perp}} \frac{\partial f_{o}}{\partial v_{\perp}}\right) \\
& \psi=\frac{\omega_{p i}^{2} \Omega^{3}}{c^{2} k_{\perp}^{4}} \sum_{n, n} \int_{d} v_{\sim}^{\prime} d v_{\perp} \int_{-\infty}^{\infty} \frac{d v_{\|} d v_{\|}^{\prime} J_{n}^{2}(w) J_{n}^{2}\left(w^{\prime}\right)}{\left(k_{\|} v_{\|}-w+n \Omega\right)\left(k_{\|} v_{\|}^{\prime}-w+n \Omega\right)} \\
& \dot{n}^{\prime}\left(v_{\|}-v_{\|}^{\prime}\right)\left\{\left[\omega \frac{\partial f_{0}}{\partial v_{\|}}-n \Omega\left(\frac{\partial f_{0}}{\partial v_{\|}}-\frac{v_{\|}}{v_{\perp}} \frac{\partial f_{0}}{\partial v_{\perp}}\right)-k_{\|} v_{\|}^{\prime} \frac{\partial f_{0}}{\partial v_{\|}}\right] \frac{1}{v_{\perp}^{\prime}} \frac{\partial f_{0}}{\partial v_{\perp}^{\prime}}\right. \\
& \left.+k_{\|} \frac{\partial f_{0}}{\partial v_{\|}} \frac{\partial E_{0}}{\partial v_{\|}}\right\}
\end{aligned}
$$

Further simplifications of $\Psi$ appear possible only in the special cases discussed below. Note that as $k_{1}^{2} \rightarrow 0$, both $\Theta$ and $\psi$ remain finite.

Equation (17) is the dispersion relation on which our subsequent discussions are based. 


\section{INSTABILITIES OF STRONG BEAMS}

A strong beam for the purposes of this section is one in which the concentration of cold background ions is small compared to the concentration, of beam ions, and we shall ignore the background ions. Strong beams are subject to both purely growing and overstable modes ${ }^{7}$ with $\left(\dot{\omega} \approx \mathrm{k}_{\|} \mathrm{U} \approx \Omega / 2\right)$. We shall consider these cases separately:

Turning first to the purely growing modes, one can verify that the most important terms in (18) and (19) involve only $\partial / \partial v_{\perp}$ with the result that

$$
\begin{aligned}
& \Theta=-\sum_{n} \frac{\Omega^{2}}{k_{i}^{2}} \int_{1 !} d v_{\perp} \int_{-\infty}^{\infty} \cdots \frac{J_{n}^{2}(w) n \Omega}{k_{\|\|} v_{\|}-w+n \Omega} \frac{\partial f_{o}}{v_{\perp} \partial v_{\perp}} d v_{\|} \\
& \quad \therefore \quad=\sum_{n} \therefore \frac{1}{4} \Gamma_{n} \frac{n \Omega}{k_{\|} \bar{v}_{b}}\left(z_{n}^{(+)}+z_{n}^{(-)}\right)
\end{aligned}
$$

where

$$
\begin{aligned}
& \Gamma_{n}=\frac{2 I_{n}(\lambda)}{\lambda} e^{-\lambda} ;:|n| \geqq 1 \\
& \Gamma_{n}=\Gamma_{0}=I_{0}(\lambda) e^{-\lambda} ; n=0 \\
& \lambda=\frac{k_{\perp}^{2} T_{\perp}}{M \Omega^{2}} \ldots
\end{aligned}
$$


and

$$
\mathrm{z}_{\mathrm{n}}^{( \pm)}=\mathrm{z}\left(\frac{\omega-\mathrm{n} \Omega \pm \mathrm{k}_{\|} \mathrm{U}}{\mathrm{k}_{\|} \overline{\mathrm{v}}_{\mathrm{b}}}\right), \overline{\mathrm{v}}_{\mathrm{b}}=\left(\frac{2 \mathrm{~T}}{\mathrm{M}}\right)^{1 / 2}
$$

We shall consider cases where $k_{\|} \mathrm{U} \approx \Omega$ so that only in terms with $\mathrm{n}= \pm 1$ is the complete dispersion function required; in the rest the asymptotic expansions can be used. Next, introduce the real functions $\mathrm{R}$ and $\mathrm{I}$ such that

$$
R=\operatorname{Rez}\left(\frac{k_{1} U-\Omega}{k_{\|} \bar{v}_{b}}\right) \quad I=\operatorname{Imz}\left(\frac{k_{1} U-\Omega}{k_{\|} \bar{v}_{b}}\right)
$$

Using the fact the $R$ is an odd function while $I$ is even, one finds that

$$
\Theta=\frac{\Gamma_{1}}{2}\left(\frac{\Omega}{k_{\|} \bar{v}_{b}} R+\frac{\Omega}{\Omega+k \| U}\right)+\Delta(\lambda)+\sum_{n=2}^{\infty} \frac{\Gamma_{n}}{\lambda}\left(\frac{k_{1}^{2} U^{2}}{n^{2} \Omega^{2}-k_{\|}^{2} U^{2}}\right)
$$

where

$$
\Delta(\lambda)=\frac{1}{\lambda}\left(1-\Gamma_{0}-\lambda \Gamma_{1}\right)
$$

The power series expression for $\Delta$ is

$$
\Delta=\frac{\lambda}{4}-\frac{5 \lambda^{2}}{24}+\frac{7 \lambda^{3}}{64}+\ldots \ldots
$$

and $\Delta$ achieves a maximum value of $\Delta \simeq .13$ : when $\lambda \approx 1.5$. We shall confine our attention to moderate values of $\lambda(i . e \cdot \lambda<2)$ where the summation in (24) can be neglected. 
When only terms in $\partial / \partial v_{1}, \partial / \partial v_{1}^{\prime}$ are retained in expression (19) for. $\psi$, one finds that

$$
\begin{aligned}
& \Psi=\frac{\omega_{p i}^{2}}{c^{2}} \frac{\Omega^{4}}{k_{\perp}^{4}} \sum_{n, n} \int d v_{\perp}^{\prime} d v_{\perp} \int_{-\infty}^{\infty} \frac{d v_{\|} d v_{\|}^{\prime} J_{n}^{2}(\omega) J_{n}^{\prime 2}(\omega)}{\left(k_{\|} v_{\|}-\omega+n \Omega\right)\left(k_{\|} v_{\|}-\omega+n \Omega\right)} \\
& \frac{n n^{\prime}\left(v_{\|}-v^{\prime}\right)^{2}}{2} \frac{1}{v_{\perp}} \frac{\partial f}{\partial v_{\perp}} \frac{1}{v_{\perp}^{\prime}} \frac{\partial f}{\partial v_{\perp}^{\prime}}
\end{aligned}
$$

Utilization of the same approximations for $\Psi$ results in

$$
\Psi=\frac{\mathrm{U}^{2}}{\mathrm{v}_{\mathrm{A}}^{2}}\left\{\left[\frac{\Gamma_{1}}{2}\left(\frac{\Omega}{\mathrm{k}_{\|} \overline{\mathrm{v}}_{\mathrm{b}}} \mathrm{R}+\frac{\Omega}{\Omega+\mathrm{k}_{\|} \mathrm{U}}\right)+\Delta\right]^{2}+\frac{\Gamma_{1}^{2}}{4} \frac{\Omega^{2}}{\mathrm{k}_{\|}^{2-2} \mathrm{v}_{\mathrm{b}}^{2}} \mathrm{I}^{2}\right\}
$$

Also, in the context of this approximation, one can ignore $\mathrm{K}_{\mathrm{zz}}^{0}$ provided $U^{2} \gg T_{e} / M$ and (4) simplifies to

$$
\frac{\omega^{2} \mathrm{k} x \mathrm{x}}{\mathrm{c}^{2} \mathrm{k}_{\|}^{2}}=\frac{\Omega^{2}}{\mathrm{k}_{\|}^{2} \mathrm{v}_{\mathrm{A}}^{2}}\left(\frac{\mathrm{ur} \mathrm{I}}{2 \overline{\mathrm{v}}_{\mathrm{b}}} \mathrm{R}-\frac{\Gamma_{I}}{2} \frac{\mathrm{k}_{\|} \|^{\mathrm{U}}}{\mathrm{k}_{\|} \mathrm{U}+\Omega}+\frac{\mathrm{k}_{1}^{2} \mathrm{U}^{2}}{\Omega^{2}} \Delta\right)
$$

The instabilities will have their minimum threshold when

$$
k_{\|} \mathrm{u} \approx \Omega
$$

so that upon neglecting terms small by the ratio $\bar{v}_{b} / U$ compared to $R$; the dispersion relation becomes 


$$
\begin{aligned}
& \frac{\mathrm{k}_{\perp}^{2}{ }^{2}{ }_{\mathrm{p} i}^{2}}{\Omega^{2}}\left\{\frac{\Gamma_{1}}{2} \frac{\mathrm{U}}{\overline{\mathrm{v}}_{\mathrm{b}}} \mathrm{R}+\Delta-\frac{\mathrm{U}^{2}}{\mathrm{v}_{\mathrm{A}}^{2}}\left[\left(\frac{\Gamma_{\mathrm{I}}}{2 \overline{\mathrm{v}}_{\mathrm{b}}} \mathrm{R}+\Delta\right)^{2}+\frac{\Gamma_{1}^{2} \mathrm{U}^{2}}{\left.\left.4 \overline{\mathrm{v}}_{\mathrm{b}}^{2} I^{2}\right]\right\}}\right.\right. \\
& =\frac{1}{\mathrm{D}_{\mathrm{e}}^{2}}\left[\frac{\mathrm{U}^{2}}{\mathrm{v}_{\mathrm{A}}^{2}} \cdot\left(\frac{\mathrm{U}}{\overline{\mathrm{v}}_{\mathrm{b}}} \cdot \frac{\Gamma_{1}}{2} \mathrm{R}+\Delta\right)-1\right]
\end{aligned}
$$

When $v_{A}^{2} \rightarrow \infty$, the electrostatic dispersion relation of Weibel ${ }^{7}$ is recovered. Dispersion Relation (28) can be recast into

$$
\begin{aligned}
& \frac{-\mathrm{k}_{\perp}^{2} \mathrm{~T} e}{\mathrm{M} \Omega^{2}} \frac{\Gamma_{1}^{2} \mathrm{U}^{4}}{4 \mathrm{~V}_{\mathrm{A}}^{2} \overline{\mathrm{v}}_{\mathrm{b}}^{2}} \mathrm{I}^{2}=\left[1+\frac{\mathrm{k}_{\perp}^{2} \mathrm{~T} e}{\mathrm{M} \Omega^{2}}\left(\frac{\Gamma_{1} \mathrm{U}}{2 \overline{\mathrm{v}}_{\mathrm{b}}} \mathrm{R}+\Delta\right)\right] \\
& \cdot \quad\left[\frac{\mathrm{U}^{2}}{\mathrm{v}_{\mathrm{A}}^{2}}\left(\frac{\Gamma_{1}}{2 \overline{\mathrm{v}}_{\mathrm{b}}{ }^{2}} \mathrm{R}+\Delta\right)-1\right]
\end{aligned}
$$

from which it is clear that electromagnetic modes will be unstable whenever

$$
\mathrm{U}^{3}>2 \mathrm{v}_{\mathrm{A}}^{2} \overline{\mathrm{v}}_{\mathrm{b}} / \mathrm{R}_{\max }
$$

where $R_{\max }=1.08 \approx 1$. Defining the Alfven energy $E_{A}=M V_{A}^{2} / 2$, the beam energy $E_{b}=M U^{2} / 2$, and beam energy spread $\Delta E_{b}=2 M U \bar{v}_{b}$, one can rewrite. (30)

$$
E_{b}^{2}>\frac{E_{A} \Delta E_{b}}{2}
$$

The electrostatic modes become unstable when the first [] brackets on the right-hand side of (29) vanish. Recognizing that

$$
\begin{gathered}
\max _{\perp}\left(\frac{\mathrm{k}_{\perp}^{2} \mathrm{~T}}{\mathrm{M} \Omega^{2}} \Gamma_{\mathrm{i}}\right) \approx 0.4 \frac{\mathrm{T}}{\mathrm{T}} \\
\lambda \Delta \approx 0.1
\end{gathered}
$$


one recovers Weibel's electrostatic result instability criterion

$$
\frac{U}{\bar{v}_{b}}>5\left(\frac{T_{\perp}}{T_{e}}+0.1\right)
$$

or equivalently

$$
E_{b}>\frac{5}{4}\left(\frac{T_{\perp}}{T_{e}}+0.1\right) \Delta E_{b}
$$

Another electromagnetic marginal stability point exists when $\mathrm{R}=0, \mathrm{I}=\sqrt{\pi} \cdot$ Utilizing

$$
\max _{1}\left(\frac{\mathrm{k}_{\perp}^{2} \mathrm{~T} e}{\mathrm{M} \Omega^{2}} \Gamma_{1}^{2}\right) \approx \frac{\mathrm{T}_{\mathrm{e}}}{6 \mathrm{~T}_{\perp}}
$$

one finds the instability criterion

$$
\frac{\pi}{24} \frac{\mathrm{T}}{\mathrm{T}_{\perp}} \frac{\mathrm{U}^{4}}{\mathrm{v}_{\mathrm{A}}^{2} \overrightarrow{\mathrm{v}}_{\mathrm{b}}^{2}}>1 ; \quad \mathrm{E}_{\mathrm{b}}^{3}>\frac{3}{2 \pi} \frac{\mathrm{T}_{\perp}}{\mathrm{T}_{\mathrm{e}}} \mathrm{E}_{\mathrm{A}}\left(\Delta \mathrm{E}_{\mathrm{b}}\right)^{2}
$$

Combining these instability criteria, one can find the least stringent stability criterion. Table I gives the results: 
Table.I. Purelý Growing Instability Criteria ${ }^{\dagger}$

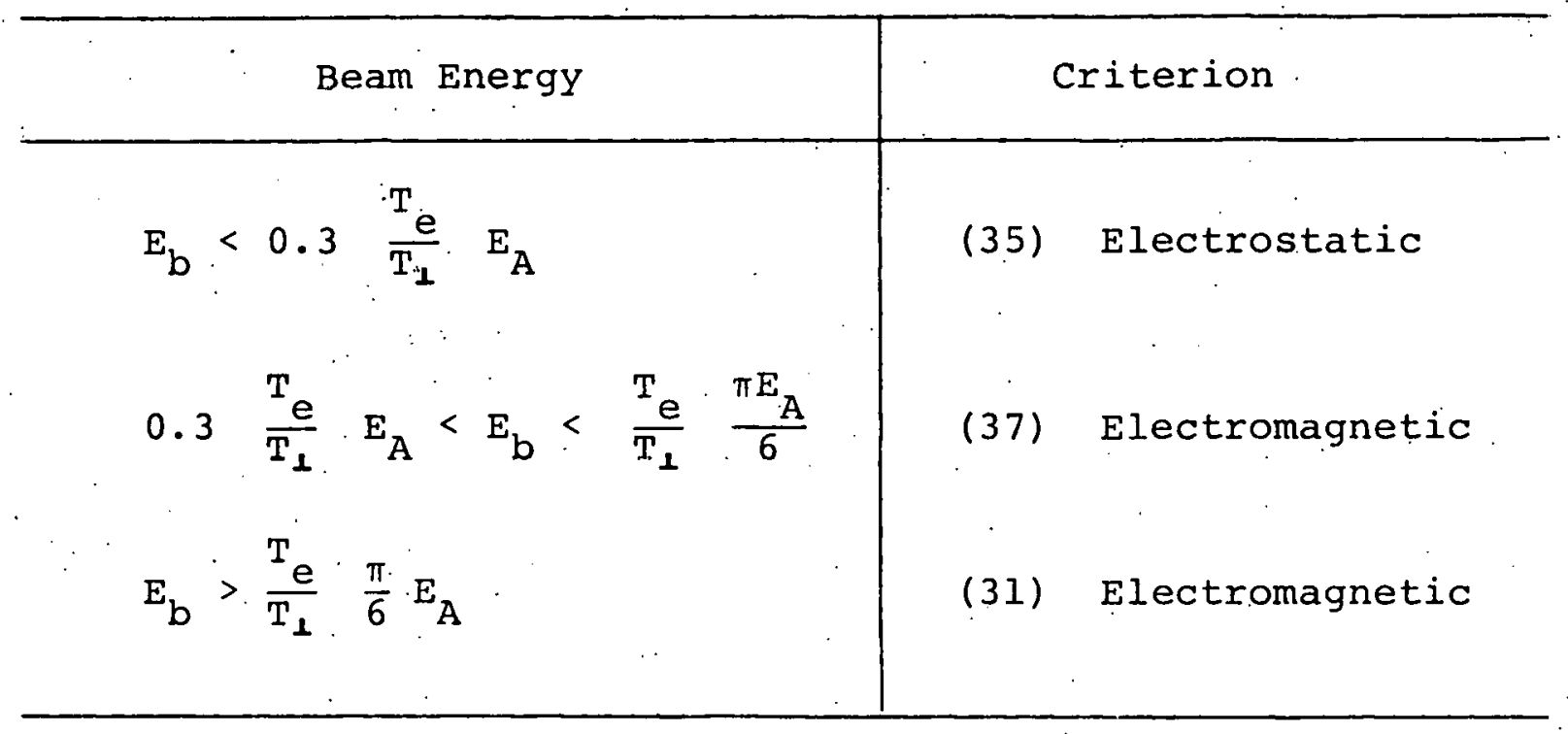

tFor conciseness, the small value of $\lambda \Delta$ appearing in $(34,35)$ has been ignored in this table.

It is evident that purely growing instabilities can occur with quite appreciable beam energy spreads.

Let us now. turn to the investigation of overstable modes with $k_{\|} \mathrm{U} \approx \omega \approx \Omega / 2$. Introducing the frequency $\omega{ }^{\prime}$

$$
\omega=k_{\|} \mathrm{u}+\omega^{\prime}
$$

one can evaluate the various terms in the dispersion relation (18): Specifically; from (18), one finds

$$
A=\frac{\Gamma_{1}}{4} \frac{\Omega}{k_{\|} \bar{v}_{b}} 7\left(\frac{\omega^{\prime}+2 k_{\|} u-\Omega}{k_{\|} \bar{v}_{b}}\right)+\frac{5}{8} \Gamma_{\perp}+\Delta
$$


while from (25) one derives

$$
\Psi=\frac{U^{2}}{2 v_{A}^{2}}\left(\frac{1-\Gamma_{0}}{\lambda}\right)\left\{\Gamma_{1}\left[\frac{\Omega}{k_{j} \bar{v}_{b}} z\left(\frac{\omega^{\prime}+2 k_{j \mid} U-\Omega}{k_{j} \bar{v}_{b}}\right)+\frac{1}{2}\right]+2 \Delta\right\}
$$

from (4)

$$
\frac{\omega^{2}}{c^{2}} k_{x x}=\frac{\omega_{p i}^{2}}{c^{2}}\left\{\frac{\Gamma_{1}}{2}\left[\frac{U}{\nabla_{b}} z\left(\frac{\omega^{\prime}+2 k_{1} U-\Omega}{k_{\|} \bar{v}_{b}}\right)-\frac{1}{4}\right]-\Delta\right\}
$$

and from (II)

$$
\mathrm{K}_{z z}^{(0)}=\frac{1}{2 \mathrm{k}_{\|}^{2} \mathrm{D}_{i}^{2}} \Gamma_{0} \mathrm{Y}_{\circ}\left(\frac{\omega}{\mathrm{k}_{\|} \overline{\mathrm{v}}_{i}}\right)
$$

When the ratio $\mathrm{u} / \overline{\mathrm{v}}_{\mathrm{b}}=4 \mathrm{E}_{\mathrm{b}} / \Delta \mathrm{E}_{\mathrm{b}}$ is large, terms not containing $\mathrm{z}$ in $(39-41)$ are negligable. One can then collect the results (39-42), incorporate them into (18), and produce the dispersion relation

$$
\begin{gathered}
\frac{\mathrm{k}_{\perp}^{2} \mathrm{~T}_{\mathrm{e}}}{\mathrm{M} \Omega^{2}} \frac{\Gamma_{1}}{4} \frac{\Omega}{\mathrm{k}_{\|} \overline{\mathrm{v}}_{\mathrm{b}}}\left(1-\frac{2 \mathrm{I}_{1} \mathrm{U}^{2}}{\mathrm{v}_{\mathrm{A}}^{2}}\right) \mathrm{z} \\
=\left[1+\frac{\mathrm{T}^{\mathrm{T}} \mathrm{o}}{2 \mathrm{~T} \|} \mathrm{Y}_{\mathrm{o}}\left(\frac{\omega}{\mathrm{k}_{\|} \overline{\mathrm{v}}_{\mathrm{b}}}\right)\right] \cdot\left(\frac{\omega_{\mathrm{pi}}^{2} \Gamma_{1} \mathrm{U}}{\mathrm{c}^{2} \mathrm{k}_{\|}^{2}{ }^{2} \overline{\mathrm{v}}_{\mathrm{b}}} \mathrm{z}-1\right)
\end{gathered}
$$

where the argument of $\mathrm{z}$ is understood to be that occurring in (39-41). Also we shall use the relationship $k_{1} \mathrm{U} \approx \Omega / 2$ and recast the dispersion relation into the form 


$$
n z=s(\Lambda z-1)
$$

where

$$
\begin{gathered}
n=\frac{k_{\perp}^{2} T_{e} \Gamma_{1} U^{2}}{2 \Omega^{2} \cdot \bar{v}_{b}}\left(1-\frac{2 \Gamma_{I} U^{2}}{v_{A}^{2}}\right) ; \Lambda=\frac{2 U^{3} \Gamma_{1}}{v_{A}^{2} \bar{v}_{b}} \\
\quad S\left(\omega^{\prime}\right)=1+\frac{T_{e}}{2 T_{\|}} \cdot Y_{O}\left(\frac{2 \omega^{\prime} U}{\Omega \bar{v}_{b}}\right) .
\end{gathered}
$$

Equation (44) is a complex marginal stability equation which must be solved for $n$ and $\lambda$ (i.e., $k_{\perp}^{2}$ and $U$ ) for a given value of $k_{\|}$, $\omega^{\prime}$. One must then minimize the value of $U$ with respect to $k_{\perp}, \omega^{\prime}, k_{\|}$; Note that $\omega^{\prime}$ 'determines the value of $s$ whereas, for a fixed $\omega$, the value of $z$ can be changed by small relative changes in $k_{\|}$. Letting $k_{\|_{0}}{ }^{j} \equiv \Omega / 2, k_{\|}=k_{\|_{0}}+\delta k_{\|}$, one sees that

$$
z\left(\frac{\omega^{\prime}+2 \mathrm{k}_{\|} \mathrm{u}-\Omega}{\mathrm{k}_{1} \overline{\mathrm{v}}_{\mathrm{b}}}\right)=\mathrm{z}\left[\frac{2 \mathrm{U}}{\mathrm{v}_{\mathrm{b}}}\left(\frac{\omega^{\prime}}{\Omega}+\frac{\delta \mathrm{k}_{1}}{\mathrm{k}_{\|_{\mathrm{o}}}}\right)\right]
$$


The arguments of $S$ and $z$ are effectively independent. One can solve (44) for $\eta$ and $\Lambda$ with the result

$$
\begin{aligned}
\Lambda & \because \frac{\operatorname{Re}(\mathrm{Z}) \operatorname{Im}(\mathrm{S})-\operatorname{Re}(\mathrm{S}) \operatorname{Im}(\mathrm{Z})}{|\mathrm{z}|^{2} \operatorname{Im}(\mathrm{S})} \\
& \therefore \quad \\
& \therefore \quad n=-\frac{\operatorname{Im}(\mathrm{z})|\mathrm{S}|^{2}}{|\mathrm{z}|^{2} \operatorname{Im}(\mathrm{S})} .
\end{aligned}
$$

One should also note that the ratio $\mathrm{T}_{\mathrm{e}} / \mathrm{T}_{\|}$may be large. This is particularly clear when it is expressed in terms of the beam energy spread, $\Delta \mathrm{E}_{\mathrm{b}}=2 \mathrm{MU}\left(2 \mathrm{~T}_{\mid} / \mathrm{M}\right)^{1 / 2}$; we find that

$$
\frac{T_{e}}{2 T_{\|}}=\frac{8 T_{e} E_{b}}{\left(\Delta E_{b}\right)^{2}} \equiv \tau
$$

Three conditions must" he satisfied if instability is to occur.

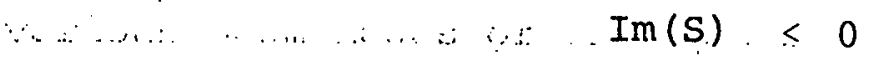

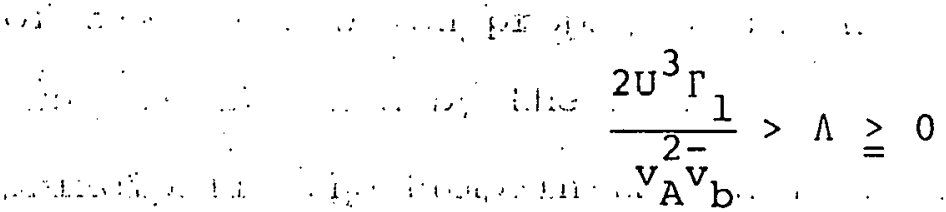

$$
\therefore \ldots \lambda \Gamma_{1} \frac{T_{e}}{2 T_{\perp}} \frac{U}{\bar{v}_{b}}\left(1-\frac{2 \Gamma_{1} U^{2}}{v_{A}^{2}}\right)>n \text {. }
$$


When $\Lambda=0$, the electrostatic instability condition results. These conditions differ from Weibel's ${ }^{7}$ treatment in two respects. First, Weibel does not keep imaginary contributions to $\mathrm{s}$ or $\mathrm{z}$ so that only a real equation results. Second, the use of his approximation (4) for the $\mathrm{n}=0$ ion terms discards possibility of a beam-acoustic mode and concomitantly, the instability qenerated by the interaction between the slow beam-acoustic mode and the cyclotron wave. Consequently, our instability condition combining $(49 \mathrm{a}, 51)$ and $\mathrm{Fig}$. $2 \mathrm{a}$ results in a lower threshold than Weibel, cspecially if $\tau>1$.

In terms of the beam energy and energy spread, the instability criteria are

$$
\begin{gathered}
E_{b}^{2}>E_{A} \Delta E_{b}\left(\frac{\Lambda}{8 \Gamma_{1}}\right) \\
E_{b}\left(1-\frac{2 \Gamma_{1} E_{b}}{E_{A}}\right)>\left(\frac{\eta}{2 \lambda \Gamma}\right)\left(\frac{T_{\perp}}{T_{e}}\right) \Delta E_{b} .
\end{gathered}
$$

Figure 2a presents the values of $n$ appiupriate to electrostatic instabilities $(\Lambda=0)$ as a function of lhe ratio $\tau$ defined in (48). This criterion differs from the purely growing electrostatic criterion. (33) when $n$ differs from unity. Clearly, Fig. 2 shows that when $\tau$ is large, the overstable criterion (5l) is less stringent. 
The electromagnetic instability criteria (50-51) are most easily satisfied if' $n$ 'is small, so they differ from the electrostatic case only when $\tau \dot{\sim} 1$ : Figure $2 \mathrm{~b}$ graphs the minimum values of $\Lambda$ vs. $\tau$ for a sequence of values of $n$. Note that $\Lambda$ always has values $\Lambda .<4$, which means that the overstable marginal stability criterion is less stringent than the purely growing criterion (31).

The principal conclusion of this section is that the strong beam instability criterion is governed principally by the relative energy spread of the beam, and is not stabilized by $\mathrm{T}_{e} / \mathrm{T}_{\perp}<2$ as was the case with the field-free theory. Modes with $\omega \approx \Omega / 2$ are most easily destabilized.

\section{WEAK BEAM INSTABILITIES}

Many research and thermonuclear plasmas contain ion beams whose concentration are small compared to the background ion species. In such circumstances, one can generally ignore the various beam modes of the system and concentrate on the stability of the waves which propagate in the background plasma and which are destabilized, by the beam-free energy in velocity space-principally the bump-in-the-tail character of the ion velocity distribution. Denoting by $\varepsilon$ the relative concentration of beam to background plasmas 


$$
\dot{\varepsilon} \equiv \mathrm{n}_{\mathrm{b}} / \mathrm{n}_{\mathrm{c}} \approx \mathrm{n}_{\mathrm{b}} / \mathrm{n}_{\mathrm{e}}
$$

one can evaluate the terms entering into dispersion relation (17). Let us focus on the instabilities generated by the interaction between the ion beam and cyclotron waves, either electromagnetic or electrostatic, i.e.,

$$
\omega \approx \mathrm{k}_{\|} \mathrm{U} \approx \Omega^{\circ}
$$

The other possibility of a "backward" cyclotron resonance with beam requires a higher beam velocity $U=(\omega+\Omega) / k_{\|}$and so does not determine the minimum drift velocity necessary for instability. $\therefore$ Our work on strong beams has shown that the contribution of Bessel functions with order. $n \geqq 2$ is small. Hence we retain only $|n|=1$ terms in (18) and find

$$
\theta=\frac{\Gamma_{\mathrm{c}}}{2}\left[\frac{\Omega}{\mathrm{k}_{\|} \overline{\mathrm{v}}_{\mathrm{C}}} \mathrm{z}\left(\frac{\omega-\Omega}{\mathrm{k}_{\|} \overline{\mathrm{v}}_{\mathrm{C}}}\right)+\frac{1}{\omega+\Omega}\right]
$$

where

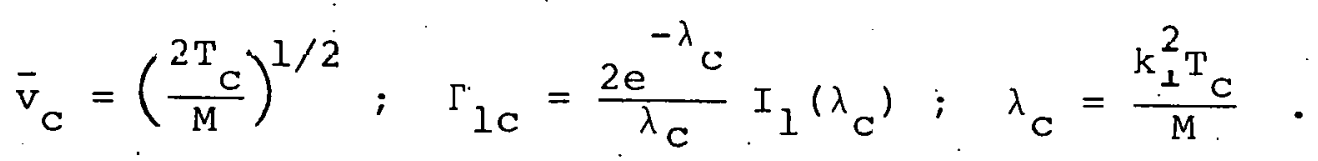


Only the background ions contribute to $\Psi$ and $\mathrm{K}_{\mathrm{xx}}$ because $\mathrm{n}, \mathrm{n}{ }^{\prime}=0$ resonances are not included in the sum. Because the background ions are assumed isotropic, many terms cancel in (16) and one finds (again retaining only $|n|=\left|n^{\prime}\right|=1$ terms)

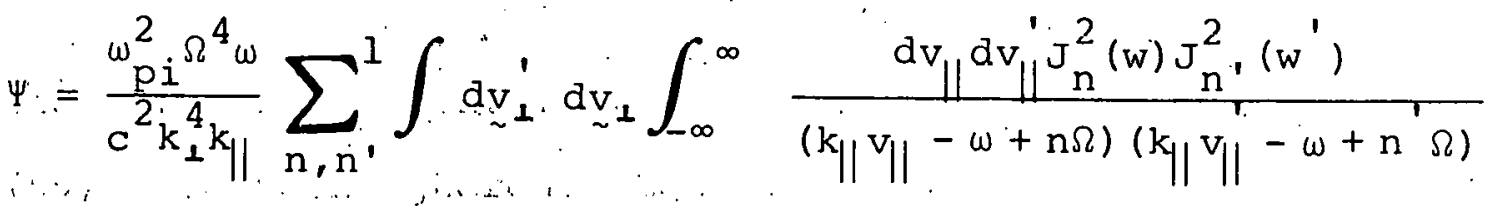

$$
\begin{aligned}
& \because n_{1} n^{\prime}\left(n-n^{\prime}\right) \frac{\partial f_{0}}{\partial v_{\|}} \frac{i}{v_{1}^{\prime}} \frac{\partial f_{0}}{\partial v_{1}^{\prime}} \\
& \therefore=\frac{\Gamma^{2}}{2}\left[\frac{\mathrm{u}^{2}}{\mathrm{v}_{\mathrm{A}}^{2}} \frac{\Omega^{2}}{\omega(\omega+\Omega)} \mathrm{Y}_{\mathrm{O}}\left(\frac{\omega-\Omega}{\mathrm{k}_{\|} \overline{\mathrm{v}}_{\mathrm{C}}}\right)\right. \\
& \left.+\frac{U}{\bar{v}_{c}} \beta_{c} \frac{\Omega^{2}}{(\omega+\Omega)^{2}} z\left(\frac{\omega-\Omega}{k_{\|} \bar{v}_{c}}\right)\right]
\end{aligned}
$$

and

$$
\frac{\omega^{2}}{\mathrm{c}^{2} \mathrm{k}_{\|}^{2}} \mathrm{~K}_{\mathrm{xx}}=\frac{\Gamma_{i}}{2} \frac{\omega_{\mathrm{p} i}^{2}}{\mathrm{c}^{2} \mathrm{k}_{\|}^{2}}\left[\frac{\omega}{\mathrm{k}_{\|} \overline{\mathrm{v}}_{\mathrm{c}}} \mathrm{z}\left(\frac{\omega-\Omega}{\mathrm{k}_{\|} \overline{\mathrm{v}}_{\mathrm{c}}}\right)-\frac{\omega}{\omega+\Omega}\right] .
$$

The smali concentration of beam ions permits one to ignore the real part of $\mathrm{K}_{\mathrm{zz}}^{(0)}$ relative to the contribution of the electrons. and obtain

$$
\frac{1}{D_{e}^{2}} Y_{0}^{(e)}+k_{\|}^{2} K_{z z}^{(0)}=\frac{1}{D_{e}^{2}}\left[1+\frac{i \sqrt{\pi} \omega}{k_{\|} \bar{v}_{e}}-\frac{i \pi T_{e} \varepsilon}{M} \Gamma_{0}\left(\frac{\partial f_{b}}{\partial v_{\|}}\right)_{\cdot v_{\|}=\omega / k_{\|}}\right]
$$


The results of Eqs. $(53-57)$ can now be combined into (17) to form the weak beam dispersion relation. This equation can be simplified by noting that according to (53) and (55) $\psi$ is negligible compared to $\theta$. Furthermore, instabilities will be favored when the frequency is not precisely equal to the cyclotron frequency (thus avoiding cyclotron damping by the background ions) and we can use the asymptotic expansion for $z$ :

$$
z=\frac{k_{1} \bar{v}_{C}}{\Omega-\omega}+i \sqrt{\pi} \exp \left(-\frac{M_{C}(\omega-\Omega)^{2}}{2 k_{\|^{2}{ }_{C}}}\right)
$$

Hence, the dispersion relation is

$$
\begin{aligned}
& \frac{k_{1}^{2} \omega_{p i}^{2} \Gamma_{i c}}{\omega^{2}}\left(\frac{\omega^{2}}{\Omega^{2}-\omega^{2}}+i \frac{\omega^{3}}{2 \Omega^{2} k_{\|} \bar{v}_{c}} \operatorname{Imz}\right) \\
& =\frac{1}{D_{e}^{2}}\left[I+i \sqrt{\pi} \frac{\omega}{k_{\|} \bar{v}_{e}}-i \frac{\pi e_{e}^{\varepsilon}}{M_{b}}\left(\frac{\partial f_{b}}{\partial v \|}\right) \Gamma_{0}\right] \\
& \cdot\left[\frac{\omega_{p i}^{2} i c}{k_{\|}^{2} \Gamma^{2}}\left(\frac{\omega^{2}}{\Omega^{2}-\omega^{2}}+i \frac{\omega}{2 k_{\|} \bar{v}_{c}} \operatorname{Imz}\right)-1\right] .
\end{aligned}
$$

The real part of the dispersion relation can be written two ways:

$$
\begin{aligned}
& \omega^{2}=\frac{\Omega^{2}+\left(k_{\perp}^{2} T_{e} / M\right) \Gamma_{1 c}}{I+\left(\Gamma_{\left.1 c^{2} / k_{\|} v_{A}^{2}\right)}\right.} \\
& k_{\perp}^{2}=\frac{\omega^{2}}{c^{2} k_{\|}^{2} D^{2}}(1-\sigma)
\end{aligned}
$$

or 
with $\sigma$ defined by

$$
\therefore \quad \therefore \quad \therefore \omega_{\mathrm{pi}}^{\omega^{2} \Gamma_{I c}} \quad=\frac{\mathrm{k}_{\mathrm{A}}^{2} \mathrm{c}^{2}\left(\Omega^{2}-\omega^{2}\right)}{\mathrm{U}^{2} \Gamma_{1 \mathrm{c}}} \frac{\Omega^{2}-\omega^{2}}{\Omega^{2}}
$$

where the small contributions due to the imaginary terms in (59) have been ignored. Equations $(60-61)$ show that waves will propagate in two regimes: The first regime is defined by $0<\sigma<1$ and corresponds to electromagnetic waves. The second regime occurs when $\sigma<0 \quad($ i.e., $\omega>\Omega)$ and is the regime of electrostatic waves:' Using $(60-61)$, one can evaluate the finite radius parameter to be

$$
\frac{\mathrm{k}_{\perp}^{2} \mathrm{~T}_{\mathrm{C}}}{\mathrm{M} \Omega^{2}}=\frac{\mathrm{T}_{\mathrm{C}} \mathrm{U}^{2}}{\mathrm{~T}_{\mathrm{e}} \mathrm{v}^{2}}(1-\sigma)
$$

Generally, this parameter will be small.

The imaginary part of dispersion relation (59) can be cast into the form

$$
\begin{aligned}
& \because \ldots . \quad \therefore \quad-\frac{U}{\bar{v}_{\varsigma}} \operatorname{Imz}\left(\frac{\left(\Omega^{2}-\omega^{2}\right)}{2 \Omega^{2}}\right)\left(\Gamma_{I c} \frac{\Omega^{2} U^{2}}{\omega^{2} v_{\Lambda}^{2}}+1\right) \\
& \therefore \therefore \therefore \therefore \ldots=\left[\sqrt{\pi} \frac{\mathrm{U}}{\bar{v}_{\mathrm{e}}}-\frac{\pi \mathrm{T}}{\mathrm{M}_{\mathrm{b}}}\left(\frac{\partial \mathrm{f}}{\partial \mathrm{v}_{\mathrm{b}}}\right)\right] \frac{(1-\sigma)}{\sigma}
\end{aligned}
$$

where, in terms of the beam energy, 


$$
\operatorname{Im}(z)=\sqrt{\pi} \exp \left[-\frac{M_{c} E_{b}}{M_{b} T_{c}}\left(\frac{\omega-\Omega}{\omega}\right)^{2}\right]
$$

and for most cases of interest,

$$
\Gamma_{1 c^{2} \Omega^{2} U^{2} /\left(\omega^{2} v_{A}^{2}\right) \ll i}
$$

Instability will result when the destabilization from the ion beam exceeds the stabilizing effects of electron Landau damping and ion cyclotron damping.

$$
\begin{gathered}
\frac{\pi T_{c}}{M_{b}}\left(\frac{\partial f}{\partial v_{b}}\right)_{u}>\frac{\sqrt{\pi U}}{\vec{v}_{c}}\left[\left(\frac{m T_{c}}{M_{c} T_{e}}\right)^{1 / 2}\right. \\
\left.+\frac{\sigma\left(\Omega^{2}-\omega^{2}\right)}{(I-\sigma) 2 \Omega^{2}} \exp \left(-\frac{M_{c} E_{b}}{M_{b} T_{c}} \frac{(\omega-\Omega)^{2}}{\omega^{2}}\right)\right] .
\end{gathered}
$$

Figure 3 plots the beam energy required to have equal cyclotron and electron Landau damping as a function of the frequency. Also included in Fig. 3 is the plot of the ratio of the beam energy to Alfvén energy required to make $\Gamma_{l c^{\sigma}} \sigma=1$.

In general, if the beam speed is comparable to, or somewhat lower than the Alfvén speed, it is possible to find a frequency that simultaneously is (1) close enough to $\Omega$ so that $\sigma<1$, and (2) sufficiently different from $\Omega$ so that cyclotron damping is negligible. In this case, electromagnetic instabilities 
result if the destabilization resulting from the beam overcomes electron Landau damping. If the Alfvén velocity greatly exceeds the beam velocity, then only electrostatic instabilities are possible. They are favored by a high temperature ratio $\mathrm{T}_{\mathrm{e}} / \mathrm{T}_{\mathrm{i}}$, because the maximum frequency shift

$$
\frac{\omega-\Omega}{\cdots \Omega_{1} \cdots} \approx \frac{\mathrm{T}_{\mathrm{e}}}{2 \mathrm{~T}_{\mathrm{c}}} \max _{\mathrm{k}_{\perp}}\left(\lambda_{\mathrm{c}} \Gamma_{l \mathrm{c}}\right) \approx 0.2 \frac{\mathrm{T}_{\mathrm{e}}}{\mathrm{T}_{\mathrm{c}}}
$$

depends on this ratio, and the importance of cyclotron damping is quite sensitive to the frequency shift. Figure 3 a also makes it clear that the beam energy must be rather large compared to the background ion temperature, if cyclotron damping is to be overcome.

\section{APPLICATIONS AND DISCUSSION}

The key result of this work is that instabilities generated by ion-streaming along a magnetic field can occur whenever the parallel beam energy spread $\Delta \mathrm{E}_{\mathrm{b}}$ is sufficiently narrow. For strong interpenetrating ion beams, the marginal stability criteria for overstable waves $(50-51)$ indicate that these waves onset before the purely growing modes (Table I). A comparison of instability conditions (50) and (51) demonstrates that electromagnetic instabilities dominate over electrostatic instabilities whenever the beam energy satisfies the inequality 


$$
E_{b} / E_{A}>\frac{\Lambda_{\min }}{10_{0}} \frac{T_{e}}{T_{\perp}}
$$

where $\eta_{0}$ is taken from Fig. $2 a$, and $\Lambda_{\text {min }}$ from Fig. $2 b$. In the case of weak beams, the instability criterion (66) depends on the frequency, which in turn depends on the parameter $\sigma$ (61). Electrostatic instabilities correspond to the limit

$$
\sigma=\frac{v_{A}^{2}}{U^{2} \Gamma_{1 c}} \frac{\Omega^{2}-\omega^{2}}{\Omega^{2}} \ll-1
$$

while electromagnetic instabilities occur for $0<\sigma<1$. Typically, if the beam velocity $U$ satisfies $U>0 . \dot{6} \mathrm{v}_{\mathrm{A}^{\prime}}$, then electromagnetic instabilities prevail because of their smaller ion damping, but the precise conditions depend on the background ion temperature, ion mass, etc. For the reasons detailed following (49), the electrostatic instability conditions presented here can be met with larger beam energy spreads than those predicted by.Weibel. ${ }^{7}$ our results contrast with the theory appropriate for magnetic field-free ${ }^{4-6}$ plasmas which predict stability when $T_{e} / T_{\perp}<2$, independent of the parallel energy spread. 
In addition to the beam instability results, this work points out the importance of a little-recognized wave in the ion-cyclotron frequency regime. Let us first note that the electromagnetic waves under consideration are not the usual electromagnetic ion-cyclotron presented in cold plasma theory. 12 Those waves have $E_{\|} \approx 0$, and $E_{x} \sim E_{y} \cdot$ By contrast, the waves considered here neglect $E_{y}$, and have finite values for $E_{x}$ and $E_{i j}$; they represent the extension, more precisely the mode conversion, of the cold plasma electromagnetic ion-cyclotron at densities exceeding that of the perpendicular resonance [Ref. 12 , Eq. (2-32)]. Such a wave propagates provided its parallel phase velocity $U$ satisfies $U \ll \bar{v}_{e}$. Particularly interesting is the limit "where $0<\sigma^{\prime}<{ }^{\prime} 1$, and dispersion relation $(60)$ takes on the simple, but unusual, form

$$
\therefore k_{\perp} \ldots \ldots k_{\perp}^{2}=\frac{w^{2}}{c^{2} k_{\|}^{2} D_{e}^{2}}
$$

This mode is close to an ordinary wave but propagating in a plasma that is extremely overdense. The key is the use of a hot plasma model to describe the electron contribution to $j_{\|}$. The hot plasma model changes the phase relationship between $j_{\|}$and $E_{\|}$by $180^{\circ}$ (relative to the cold plasma model) and permits propagation. "The inequality $0<\sigma \ll 1$ is most easily fulfilled when $\omega: \Omega$ and is equivalent to

$$
v_{A}^{2} \vec{v}_{C} / U^{3} \ll 1
$$


where, in this context, $U$ denotes the parallel phase velocity. Inequality 70 can be consistent with the condition that ion gyroradius effects be negligible taken from (62) provided $U>>\vec{v}_{C}$ or $\mathrm{T}_{\mathrm{e}} \gg \mathrm{T}_{\mathrm{c}} \cdot$ Both these conditions are easily met in basic laboratory research devices. To my knowledge, no laboratory experiments reporting a dispersion relation of the form (69) have appeared in the literature. And finally, let us also remark that this form of the ion-cyclotron wave plays an important role in radio frequency heating of tokamaks: 13

The experimental investigation of ion beam instabilities in basic laboratory research devices appears to be just getting underway. 2,14 It is not difficult to achieve plasma parameters where the Alfvén speed is less than the electron thermal speed. For example, consider a singly ionized argon plasma; then one can immediately compute

$$
\mathrm{v}_{\mathrm{A}}^{2} \mathrm{~m} / 2 \mathrm{~T}_{\mathrm{e}}=\mathrm{m} /\left(\mathrm{M} \beta_{\mathrm{e}}\right) \approx 3 \times 10^{-2} \mathrm{~B}_{\mathrm{kG}}^{2} / \mathrm{n}_{13} \mathrm{~T}_{\mathrm{eV}}
$$

where $\mathrm{B}_{\mathrm{kG}}$ is the magnetic field in kilogauss, $\mathrm{n}_{13}$ the density in units of $10^{13} \mathrm{~cm}^{-3}, T_{\mathrm{ev}}$ the electron temperature in electron volts. Hydrogen ion beams with a velocity comparable to the Alfvén velocity then have an energy. near $100 \mathrm{eV}$. The principal constraint is on achieving a sufficiently long plasma so that its length $l$ satisfies

$$
\ell>\frac{\Omega}{v_{A}}=\frac{c}{\omega_{p i}} \cdot 40 \text { cm. } \mathrm{n}_{13}^{-1 / 2}
$$


and convective electromagnetic instabilities have a sufficient length to grow.

It is beyond the'scope of this paper to discuss the role of cyclotron streaming instabilities in collisionless shocks ${ }^{1}$ except to note that the scale length of $c / \omega_{p i}$, and low threshold velocities make them an interesting source of turbulence.

What role will these instabilities play in two-component thermonuclear burning experiments with deuterium beams and tritium target plasmas? Both the analysis of stix $^{8}$ and Berk et al. ${ }^{9}$.conclude that in a steady-state situation the bumpin-the-tail character of the beam velocity distribution is not sufficiently strong; to overcome electron Landau damping. on the other hand, planned two-component thermonuclear burning experiments depend either partially or entirely on important transient stages: a preheat phase in which the ratio $\Gamma$ of the energy content of the injected beam to the initial electron energy content exceeds a value of $4-6$, and a concept whereby a beam is first injected at moderate energies ( $80 \mathrm{keV}$ ) and then energized to the optimum fusion reaction energy (150 kev) by major radius compression before appreciable slowing down has occurred. Stix's paper ${ }^{8}$ addresses transient situations but omits the stabilizing effects of finite ion temperature.

Let us first consider the preheat stage and let $\tau_{s}$ denote the energy slowing-down time for:ion-electron collisions:

$$
\frac{\mathrm{d}\left\langle E_{b}\right\rangle}{d T}=-\frac{\left\langle E_{b}\right\rangle}{\tau_{s}} ; \tau_{s}=\frac{3 M_{b} T^{3 / 2}}{8 \sqrt{2 \pi n e^{4} \ln \Lambda m} 1 / 2}=(6 \mathrm{~ms}) \frac{T_{k e V}}{n_{14}} \quad \frac{A_{b}}{z^{2}}
$$

Where $\left\langle E_{b}\right\rangle$ is the average ion energy, $T_{k e V}$ the electron temperature 
in units at kev, and $\mathrm{n}_{14}$ the electron density in units of $10^{14} \mathrm{~cm}^{-3}$. For early times, $t<4 \tau_{s} T_{e} / E_{o}$, the beam velocity distribution function is essentially a Maxwellian centered on the injected energy with a temperature $T_{\|}=T_{e} t / \tau_{s} \cdot$ The criterion [cf (66)] that the beam destabilization exceed the electron Landau damping then becomes

$$
\frac{\pi T_{e}}{M_{b}} \cdot \frac{\partial f}{\partial v_{\|}} \approx \frac{\sqrt{\pi}}{3} \frac{T_{e} \tau_{s}}{E_{b} \tau_{b}}>\sqrt{\pi}\left(\frac{E_{b} \Gamma}{T_{e} M_{b}}\right)^{I / 2}
$$

or

$$
\Gamma>\Gamma_{c}=\frac{3 \tau_{b}}{\tau_{s}}\left(\frac{m}{M_{b}} \cdot \frac{E_{b}^{3}}{T_{e}^{3}}\right)_{-}^{1 / 2}
$$

Where $\Gamma=4 \mathrm{n}_{\mathrm{b}} \mathrm{E}_{\mathrm{o}} / 3 \mathrm{~T} \mathrm{e}$ is proportional the beam energy content injected in a time $\tau_{b}$. Typical preheat plans envision $\tau_{b}=30 \mathrm{~ms}$, $\tau_{s}=120 \mathrm{~ms}, \mathrm{M}_{\mathrm{b}} / \mathrm{m}=3.7 .10^{3}, \mathrm{E}_{\mathrm{b}}=80 \mathrm{kev}, \mathrm{T}_{\mathrm{e}}=3 \mathrm{keV}$, resulting in $\Gamma_{\mathrm{c}}=1.7$, less than the proposed value of $\Gamma=4$. For at least a short period of time, ion velocity space instabilities will be possible. Present injection experiments ${ }^{15}$ probably will not suffer instabilities because the value of $\tau_{b} / \tau_{s}$ is larger. Une must then ask whether electromagnetic or electrostatic instabilities will prevail. The energy of a deuteron beam traveling at the Alfvén velocity in a tritium plasma is

$$
E_{A}=\frac{M_{h}}{M_{C}} \frac{B^{2}}{8 \pi n}=400 \mathrm{keV}\left(\frac{B}{50 k G}\right)^{2}\left(\frac{10^{14} \mathrm{~cm}^{-3}}{n}\right)
$$


an energy which, from Fig. 2, precludes electromagnetic instabilities for an $80 \mathrm{keV}$ beam unless the temperature of the ions is $1.2 \mathrm{keV}$ or less. Electrostatic instabilities require an initial electron-to-ion temperature ratio $\mathrm{T}_{\mathrm{e}} / \mathrm{T}_{\mathrm{C}}>3$ to permit the instability frequency to reach $\omega=1.5 \Omega$. thereby reducing the ion damping.

$\therefore$ The danger of instabilities also exists in a heating scheme where the fusion beam is energized by compression--definitely a transient situation. Using the estimate (based on a triangularshaped distribution function),

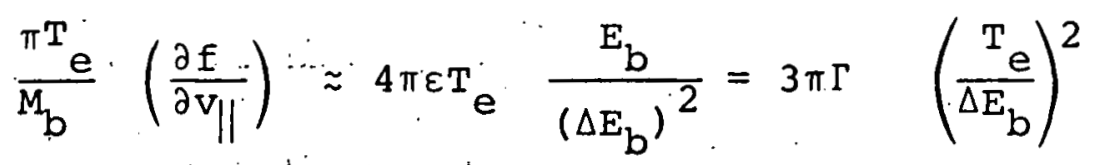

one finds ion destabilization exceeds electron Landau damping when

$$
\Gamma>\frac{1}{3 \sqrt{\pi}}\left(\frac{\mathrm{m}}{\mathrm{M}_{\mathrm{b}}}\right)^{1 / 2}\left(\frac{\mathrm{E}_{\mathrm{b}}}{\mathrm{T}_{\mathrm{e}}}\right)^{1 / 2}\left(\frac{\Delta \mathrm{E}_{\mathrm{b}}}{\mathrm{T}_{\mathrm{e}}}\right)^{2}
$$

Estimating that $E_{b}=150 \mathrm{keV}, \mathrm{T}_{\mathrm{e}}=10 \mathrm{keV}, \Delta \mathrm{E}_{\mathrm{b}}=50 \mathrm{keV}$, one finds

$$
\Gamma>\frac{1}{3}
$$

Furthermore, because: the beam energy is higher, electromagnetic instabilities will occur provided the ion temperature is below 5 keV. 'Hence; any processes that would give rise to rapid ion energy loss, either in the preheat or compression stage, so that the ion temperature is cooled and the stabilizing effect of ioncyclotron damping lost, would promote ion-cyclotron instabilities 
of the main deuterium beam, and a consequent degradation of thermonuclear burning. For example, instabilities in the preheat phase might well produce principally electron heating.

The strong beam instabilities considered here impact on a colliding beam reactor. 16 Again, transient situations appear unstable but steady-state conditions are stable because $\Delta E_{b} \sim E_{b} \ll E_{A}$ and the effective temperature ratio [see (48)] is less than unịty.

In brief, by avoiding high densities, low ion temperatures, and low magnetic flelds, two-component thermonuclear burning experiments can probably avoid ion-streaming instabilities except during the transient preheat phase. On the other hand, a central density of $2.10^{14} \mathrm{~cm}^{-3}$ which would reduce the Alfven energy to $200 \mathrm{keV}$ would appear to lead electromagnetic ion-cyclotron instabilities. Electrostatic instabilities may be suppressed by finite Larmor radius effects associated with modest perpendicular component of the injected energy.

\section{ACKNOWLEDGMENTS}

I have benefited from discussions with H. L. Berk, C. W. Horton, R. M. Kulsrud, R. Landau, M. N. Rosenbluth, and P. H. Rutherford.

This work was supported by United States Energy and Development Administration (formerly AEC) Contract E(11-1)-3073. 
REFERENCES

$l_{K}$. I. Golden, L. M. L̈inson, and S. A. Mani, Phys. Fluids 16, $2319(1973)$.

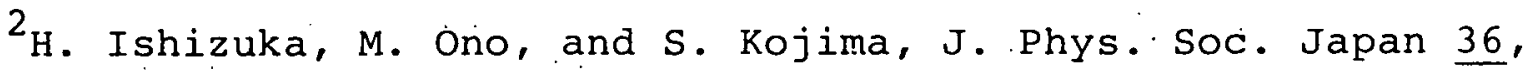
1158 (1974).

3.J. M. Dawson, H. P. Furth, and F. H. Tenney, Phys. Rev. Lett. 26, $1156 \cdot(1971)$.

${ }^{4}$ B. D. Fried and A: Y. "Wong, Shys. Fluids 9,1084 (1966).

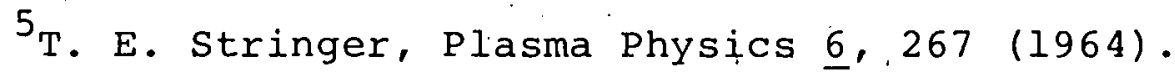

${ }^{6}$ D. W. Forslund and C. R. Shonk, Phys. Rev. Lett. 25, 281 (1970).

${ }^{7}$ E. S. Weibel, Phys. Fluids 13, 3003 (1970).

${ }^{8}$ T. H. Stix, Phys. Fluids $\underline{16}, 1922$ (1973).

${ }^{9}$ H. L. Berk, H. P. Furth, D. L. Jassby, R. M. Kulsrud, C. S. Liu, M. N. Rosenbluth, F. H. Rutherford, F. H. 'I'enney, J. L. Johnson, J. Killeen, A. A. Mirin, M. E. Resnik, and C. W. Horton, Jr., Paper IAEA-CN-33/G2-3; 5th Int. Conf. Plasma Physics and Controlled Fusion, Tokyo, (1974).

${ }^{10}$ J. M. Kindel and C. F. Kennel, J. Geophys. Res. 76, 3055, 1971; and D. W. Forslund, C. F. Kennedy, and J. M. Kindel, Current-Driven Electrostatic and Electromagnetic Ion-Cyclotron Instabilities, UCLA Plasma Physics Group Report $\$-87,1,971$.

${ }^{11}{ }_{G}$. Bekefi, Radiation Processes in Plasmas, John Wiley, New Yorkp. $229^{\circ}(1966)$. 
12.T. H. Stix, The Theory of Plasma Waves, McGraw-Hill, New York, p. 34 (1962).

${ }^{13}$ F. W. Perkins, M. Chance, and J. M: Kindel, Paper B-8, 3rd International conf. on Toroidal Plasma Confinement, Garching (1973)

${ }^{14}$ D. Grésillon and F. Doveil, Phys. Rev. Lett. 34, 77 (1975);

D. Grésilion, F. Doveil, and J. M. Buzzi, Phys. Rev. Lett. 34 , 197 (1975); N. Sato, H. Sugai, R. Hatakeyama, Phys. Rev. Lett. 34, 931 (1975).

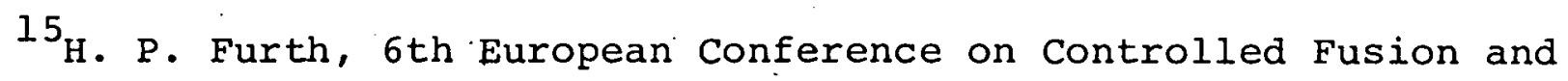
Plasma Physics, Vol. II, Invited Papers and Supplementary Papers, p.55, Moscow, 1973; K. Bol, J. L. Cecchi, C. C. Daughney, R. A. Ellis. Jr., H. P. Eubank, H. P. Furth, R. J. Goldston, H. Hsuan, R. A. Jacobsen, E. Mazzucato, R. R. Smith, and T. H. Stix, Phys. Rev. Lett. 32 , 66I (1974).

${ }^{16}$ R. M. Kulsrud, and D. L. Jassby, Neutralized Colliding-Beam Toroidal Fusion Reactors, Princeton University Plasma' Physics Laboratory Report MATT-1114 (1975). 


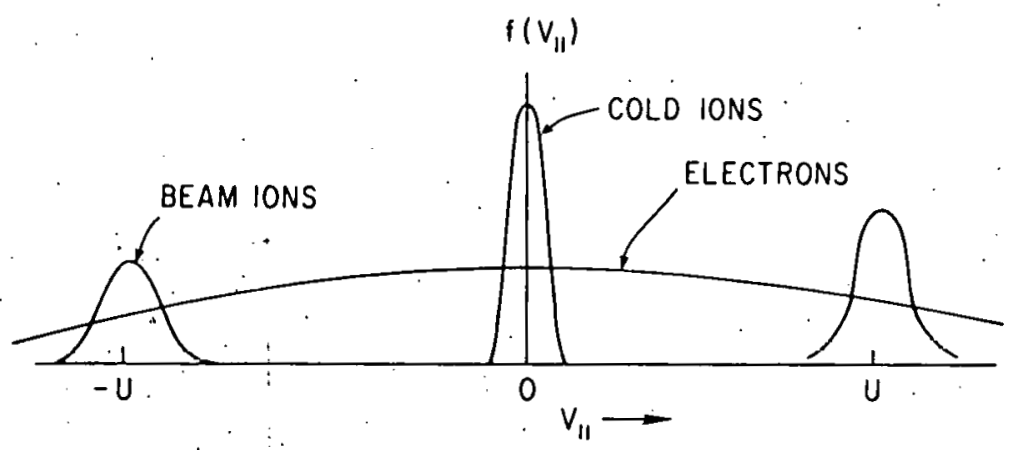

752153

Fig.1. Schematic illustration of the various parallel distribution functions. A spread in beam velocities, resulting either from a spread in injection energies, or to classical slowing down for a finite time, is modeled by a Maxwellian velocity distribution.

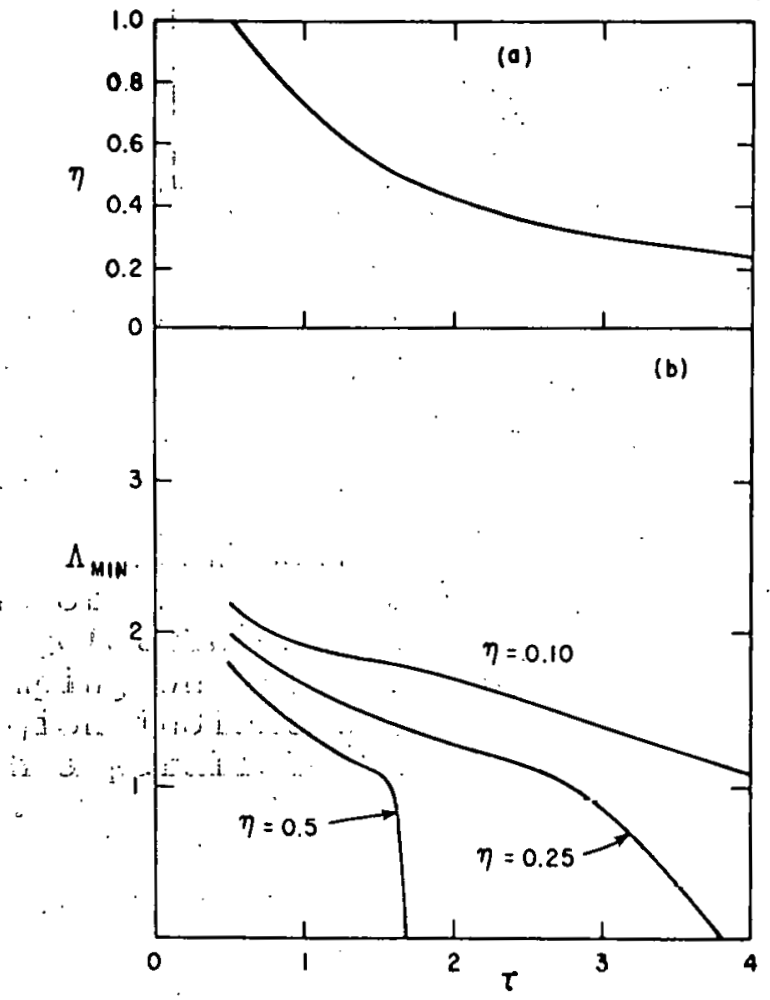

Fig: 2. Parameters governing electrostatic instabilities for the strong beam case. The quantity $\tau$ is an effective temperature ratio (48) and $\eta$ enters. into the electrostatic instability condition (51). (a) The electrostatic criterion $(\Lambda=0)$. (b) . Electromagnetic instabilities; minimum values of $\Lambda$ for fixed values of $n$. 


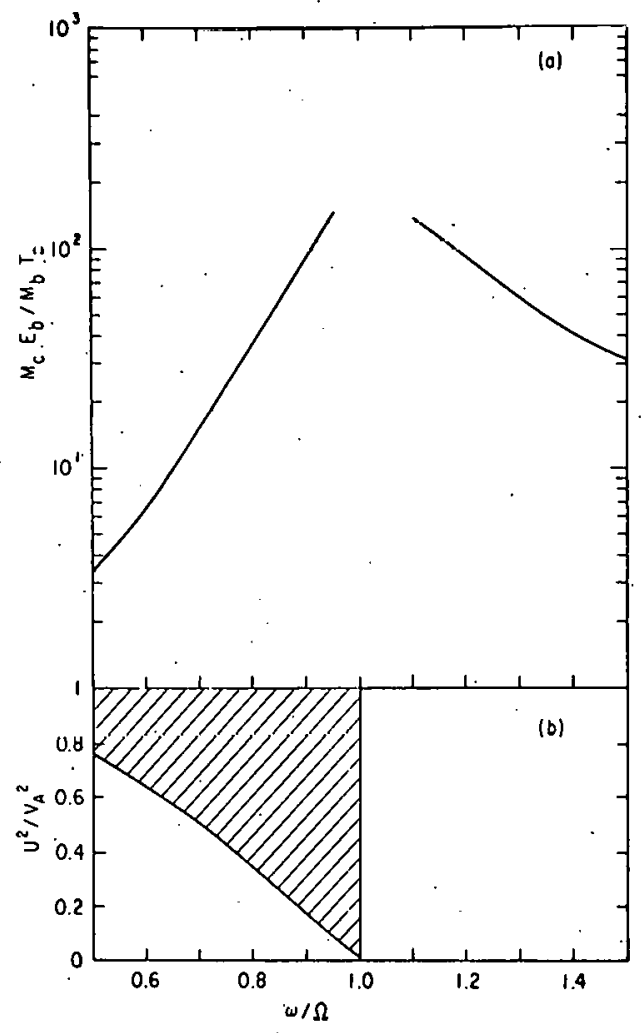

752154

Fig. 3. Weak Beam Instability Parameters. (a) Value of the quantity $M_{C} E_{b} / M_{b} T$ required to make ion-cyclotron damping equal to electron Landau damping when $|\sigma /(1-\sigma)|=1$. (b) The shaded region indicates where electromagnetic waves with a parallel phase velocity U can propagate. 
NOTICE

This report was prepared as an account of work sponsored by the United States Government. Neither the United States nor the United States Atomic Energy Commission, nor any of their employees, nor any of their contractors, subcontractors, or their employees, makes any warranty, express or implied, or assumes any legal liability or responsibility for the accuracy, completeness or usefulness of any information, apparatus, product or process disclosed, or represents that its use would not infringe privately owned rights. 